\title{
Designing a Multi-Locomotion Modular Snake Robot
}

\author{
Sara Djoudi, Jack M. Frampton, Angelina Murphy, Thomas Scammell, Toby \\ Wright, and Ze Ji[0000-0002-8968-9902] \\ School of Engineering, Cardiff University, Cardiff CF24 3AA, UK \\ jiz1@cardiff.ac.uk ${ }^{\star \star}$
}

\begin{abstract}
Snakes possess multi-locomotion abilities to best suit different environments. This work explores the design of a robot to replicate three types of snake motions: rectilinear, serpentine and sidewinding. The design featured identical modular housing units containing all the components for movement, a biomimetic skin to replicate the anisotropic friction created by the scales of the snakeskin and smart servos motors that produce adjacent housing rotation to imitate the body motion of a snake. Two prototypes are manufactured using rapid prototyping. Prototype 1 is designed to replicate rectilinear motion produced by the biomimetic snakeskin and collinear movement of each housing. Prototype 2 is powered by the smart servos and the rotation of adjacent housings to produce serpentine and sidewinding motions. From initial tests, prototype 1 is shown to be able to replicate rectilinear motion at low speeds, and prototype 2 is shown to be able to undertake 6 different movement options utilising both sidewinding and serpentine motions.
\end{abstract}

Keywords: Snake robot · biomimetic $\cdot$ kirigami $\cdot$ chain configuration

\section{Introduction}

Snake-like robots have numerous applications in an industrial context. Such examples include travelling over, or through, hazardous terrain for rescue missions [9], the development of medical equipment for stereotactic surgery due to enhanced flexion and dexterity [8] or even a role in facilitating complex tasks required by space rovers exploring extra-terrestrial environments [5]. The first snake-like robot, ACM-R5 [10], was invented in 1972. The ACM-R5 propelled forwards by twisting and turning. This robot had one degree of freedom (DoF) joints, resulting in its movement being constrained to a sine wave configuration on a flat surface simulating serpentine motion. The robot is able to travel in both water and on the ground. To allow for movement in water, paddle blades were attached around the side of the body. To aid movement on the ground, small wheels were attached to the paddle blades.

Various designs have been actively explored on robot joint configuration in recent years [5]. For example, researchers focused on developing robots with a

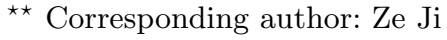


greater number of DoFs [2], enabling 3-DoF motions. The mechanism includes a moving platform and three mechanical joints: one universal joint and two composite spherical joints. Two symmetrical prismatic legs are connected to the sphere joints to replicate the snakes intercostal muscles. These joints eliminate torque, which is normal to the moving platform to protect the universal joint. To keep the original length of snake segments, the parallel joint is designed to prevent simultaneous yaw and pitch movement. The rotating axis is the cross axis of the universal joint, which entails two rotating directions perpendicular to each other.

Rectilinear motion is produced with a different principle. The scales of the skin help propel the snake forward. To replicate the behaviour of the scales, anisotropic frictional behaviour is to be simulated based on the theory in [7]. One study achieved this with nibs designed to allow for smooth and swift motion in one direction, but high friction in the opposite direction [6]. However, while a particular scale orientation would facilitate rectilinear motion perfectly, the concern is over how this might interfere with the other motion sequences our robot is intending to achieve. Another design [4] uses the art of kirigami to create a sheet of scales that buckle and pop out when the sheet is extended. During material extension, the scales pop out and contact the ground. With the scales protruded, a higher coefficient of friction with the ground in the direction opposite to the desired motion would be created. Upon retraction, this enables the elastomer to push forwards off the ground. The system is optimised to function with a pneumatic elastomer actuator.

This work aims to develop a prototype with multi-locomotion capabilities to be adaptable to its environment, allowing for effective travel across numerous terrains. We explore three main topics: modular robotics, chain robots commonly referred to as snake robots and biomimetic snakeskin. All three areas are brought together to design and manufacture a snake-like robot prototype consisting of individual identical modules connected in a chain formation capable of three types of snake motion: rectilinear, serpentine and sidewinding. A novel biomimetic snakeskin is designed and manufactured to propel the robot forwards during rectilinear motion. The modular design of the snake consists of identical housing all components necessary to produce the movement of a singular model such that they can be operated individually or linked together with inter-modular communication to form systems of varying numbers of modules. Ultimately, this would allow for greater flexibility of module configuration in the future in addition to the chain formation the current prototypes are based on.

\section{Proposed design}

\subsection{Locomotion}

Below is a summary of the three snake locomotion models to be implemented in this work. Rectilinear motion is useful for a snake travelling through tight spaces, entailing the snake moving in a straight line through contracting muscles to control the movement of the scales. The scales are alternatively lifted from 
the ground, and then pulled downward and backwards. The friction between the scales and the ground will pull the body forwards. When the scales are lifted forwards, they are stretched out creating a rougher surface in contact with the ground. This inspired us for our design. To replicate rectilinear motion, the biomimetic silicone snakeskin is developed with cuts to mimic the movement of scales using the Kirigami method. The movement of the contracting muscles to lift the scales are simulated using micro linear actuators to extend and retract the housing units. With the scales lifted, a higher coefficient of friction is created in one direction, propelling the snake forwards upon housing retraction.

Serpentine motion consists of waves of lateral bending being propagated along the body from head to tail using objects on the ground to propel itself forwards. To simulate this slithering-like motion, smart servo motors are used to instigate 180 degree rotation between adjacent housing units orthogonal to the direction of travel of the robot. When the surface is slippery, such as sand, the most common locomotion used is sidewinding, where two parts of the body are solely in contact with the ground with the remainder being held above it. The body is propelled laterally from these anchor points creating new anchor points a fixed distance away. Like serpentine motion, this movement will be replicated using servo motors to rotate the housing units. Sidewinding can be simplified by adding a vertical wave to the lateral existing wave of serpentine motion.

\subsection{Housing}

The focus for the housing design is to enable collinear and rotational movement of the housing modules and contain all components required to produce the robots motion in a compact package. Thus a 3-part module was developed, consisting of external and internal housings and an internal mounting tray.

The housing components of the module facilitated all modes of locomotion by enabling 2 modes of actuation, both within each module and between adjacent modules. The first mode of actuation is extension and retraction of the unit caused by the external housing of each module sliding over the internal housing of the same module on a fixed path (Fig. 1 (a)). This enables the rectilinear motion of the modular system when combined with the biomimetic snakeskin. The second mode of actuation is rotation of two adjacent modules facilitated by the rotation of the external housing and internal mounting tray of adjacent modules about a fixed axis with a set of brackets, (Fig. 1 (b)), to which the rotational actuator mounts.

Each of the housing parts has further functions to enable system mobility. The external module has one main purpose, ensuring that the biomimetic skin can function effectively; the smooth external surface does this by ensuring that the scales are only able to protrude in the correct direction when under extension, this was a functional priority over potential weight savings of removing material from this part (Fig. 1 (a)). The internal module facilitates the movement of the system with its slatted covering for the rotational actuator and bracket, this both protects the actuator from impact during function and gives the system a smoother surface, preventing it from catching on obstacles during 
function (Fig. 1 (b)). The internal mounting tray enables the assembly of the control systems for each module to be carried out externally of the module itself, sliding and locking into place when assembled. This facilitated easy assembly and component replacement, ensuring easy maintenance during lab testing and realworld function (Fig. 1 (c)). Fig. 2 shows a final module additively manufactured by Selective laser sintering (SLS) in Nylon.

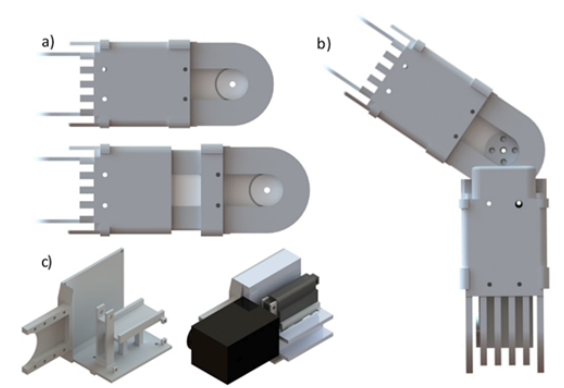

Fig. 1: CAD design of modules (a) external and internal housing in extension and retraction, (b) rotational bracket between adjacent modules, (c) internal mounting tray with and without components)

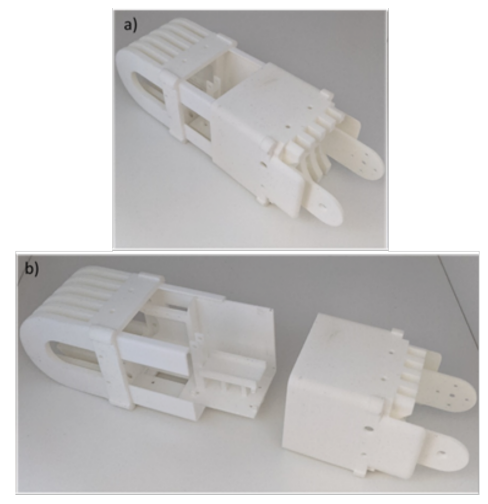

Fig. 2: Final module printed with SLS. (a) in extension, (b) an exploded view

\subsection{Smart servos}

To mimic the serpentine and sidewinding movements, servo motors are chosen due to their precise control of angular position, velocity, and acceleration. Additionally, compared to other motor types, servos have a high power-output to size and weight ratio, which is ideal for a modular-based robot. The DYNAMIXEL "smart" servos are used due to their ability to be connected in series in a daisy-chain-like formation, simplifying the electric wiring, and in-turn make the housing design more accommodating.

The motors within the body of the snake followed a coordinated sequence of movements to mimic not just the type of movement of serpentine and sidewinding, but also the direction of movement. To achieve these simulated movements, the motors followed a triangular waveform, formulated by Eq. 1:

$$
\theta(n, t)= \pm \begin{cases}V_{n}\left(t-\delta_{t}\right) & , \delta_{t} \leq t \leq \frac{T}{4}+\delta_{t} \\ -V_{n}\left(t-\delta_{t}\right)+\frac{\pi}{2}, & , \frac{T}{4}+\delta_{t} \leq t \leq \frac{3 T}{4}+\delta_{t} \\ V_{n}\left(t-\delta_{t}\right)-\pi & , \frac{3 T}{4}+\delta_{t} \leq t \leq T+\delta_{t}\end{cases}
$$

where $\theta$ is the angle of motor $n$ at time $t, v_{n}$ is the joint speed of motor $n, t$ is the elapsed time, $\delta_{t}$ is the time delay between motors, $T$ is the waveform period.

\subsection{Biomimetic Snakeskin}


The primary purpose of the skin design is to facilitate rectilinear motion through friction as demonstrated by snakeskin scales in nature [3][1]. Below is the summary of our design principle::

1. Simulating muscle propulsion to propel robot forward by the electrical actuator system

2. Creating anisotropic frictional interaction with robot's surface of travel by the skin kirigami mechanism

3. Integration of skin layer with robot body housing through strong adhesion of the skin attachment to the housing.

To simulate contracting muscles that control the movement of scales, linear actuation is used. This would extend and retract the housing, which would stretch the skin layer from its normal state. At the normal state, the housing is retracted, the skin relaxed and the scales lying flat, upon

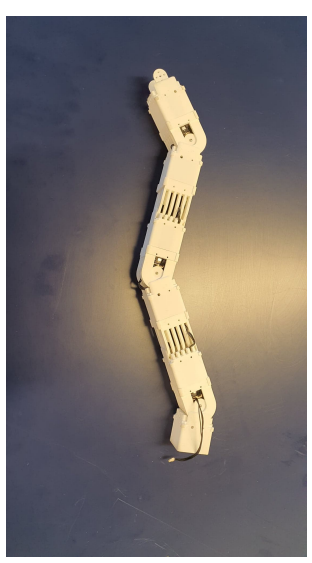

Fig. 3: Assembled prototype extension the skin stretches, and the scales protrude out. With the scales protruding, a higher coefficient of friction would be created in one direction, propelling the snake forwards upon retraction. In this project, electromechanical actuators are chosen as they do not require the extra external equipment or sealing of hydraulic and pneumatic actuators, which is difficult for the small scale of our modules.

Actuonix PQ12 Linear Actuators are chosen to be the most desirable for this project. They have a weight of $15 \mathrm{~g}$ and are capable of smooth and consistent linear stroke length of up to $20 \mathrm{~mm}$ with a load up to $50 \mathrm{~N}$. They also have an internal potentiometer, which can provide positional feedback.

Several materials were investigated and compared based on their properties. The skin material selected is silicone due to its high stiffness, good flexibility and anti-adhesive properties, so that it would minimise the coefficient of friction with the ground when flat (in the direction of motion) and not interfere with the other robotic motions.

Tension testing was conducted to establish the kirigami pattern that would yield the best performance. Several samples underwent extension under a load of $20 \mathrm{~N}$ at $8 \mathrm{~mm} / \mathrm{s}$ (as these are the peak efficiency settings of the actuator) until complete failure. Sample 1 represented the dimensions proportional to the derived equations found in the kirigami research undertaken in [4]. As for the subsequent samples, factors that were varied included adjusting the cut size, the hinge size, the angle of the cut with respect to the horizontal plane and the number of scales per row (Fig. 4).

Briefly, the following conclusions are derived from the performance results: 1 ) Cut size has minimal influence on the stiffness, 2) There is a positive correlation between hinge size and the stiffness, and 3) As the angle size increase, so does the stiffness. Ideally, the cut sizes need to be large enough to effectively protrude under the robot body weight, but not too large so as to deform and flatten 

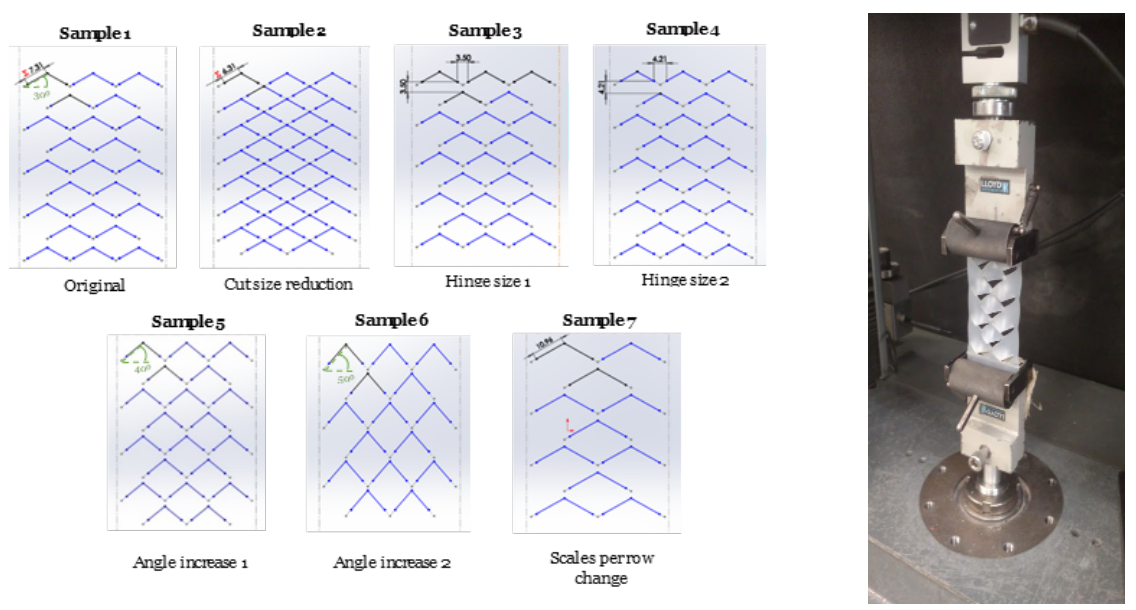

Fig. 4: Snakeskin samples designed at approximately height $=70 \mathrm{~mm}$ and width $=48 \mathrm{~mm}$ and the tension testing

against the ground surface [7]. Additionally, it is desirable for the skin to be as flexible as possible so that the layer may be extended easily by the actuator, but the skin also needs to be as stiff as possible to allow for the hinges to trigger the protrusion of the scales, without any failure propagation through the skin. From the results, sample 3 in Fig. 4 is found to be the stiffest sample that did not hinder actuator extension, and is thus selected for the robot design.

\section{Experiments and Discussion}

\subsection{Prototype 1 - Snakeskin testing}

Three tests were performed to assess the performance of the skin mechanism to extend and retract the housing and its effect on the skin layer. The areas that are examined include actuator retraction-extension motion, housing retractionextension motion and function of the scales when the skin is in tension.

Actuator with housing. The actuators were initially tested by implementing a continuous sweeping motion, causing the actuator to go from fully retracted to fully extended. Parameters of this motion were then adjusted until the actuator functioned in a smooth cyclic motion to establish the optimal values for these parameters. This code was then adjusted such that future connection of sensors to the Arduino would not be detrimental to the performance of the actuators. The actuator took 3 seconds to fully extend the housing to a $20 \mathrm{~mm}$ distance and another 3 seconds to fully retract the housing. It would have been desired for this duration to be faster. This would not cause any issues with performing rectilinear motion except it is just slower. The actuators could be swapped out for quicker ones in the future if desired.

Actuator in housing with skin attachment. This test for the skin mechanism is to test the housing extension with the skin attached to the module, in 
order prove whether the attachment can withstand the tension force indirectly produced by the actuator, and how the scales behave during the motion.

This was executed by the actuator performing a sweep motion continuously from full extension to full retraction (Fig. 5). Under full extension, the scales simultaneously protruded outwards (Fig. 5 (a)). The cuts within the skin layer created a higher coefficient of friction with the ground in the direction opposite to the desired motion. Therefore, on module retraction, the robot was able to push forwards off the ground. At full retraction, the scales flatten allowing for ease of motion (Fig. 5 (b)).

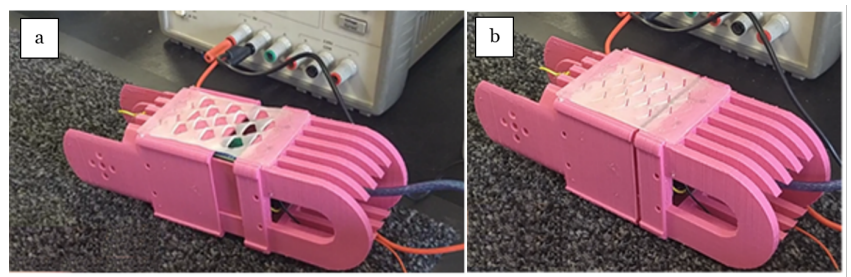

Fig. 5: Skin behaviours (a) housing extension with scales protruding, (b) housing retraction with scales flattened

Based upon the testing conducted with one housing unit, it has given a strong indication that the same mechanism could work with multiple units joined together. With more segments, the robot would have had a higher pushing force during rectilinear motion, and thus may have moved a greater distance under each cycle of the actuator extension-retraction motion.

Frictional testing. The snakeskin layer function is heavily reliant on its interaction with the surface it is travelling on. Therefore, it was imperative that frictional testing was conducted in order to quantify these interactions through obtaining the static $(\mu \mathrm{s})$ and kinetic $(\mu \mathrm{k})$ coefficients of friction between the silicone layer and various substrates.

Data was recorded for the performance of both a flat silicone sample and a scaled silicone sample to compare their interactions with various substrate surfaces. The set-up shown in Fig. 6 is employed to conduct the testing.

The flat silicone sample is attached under a $3.5 \mathrm{~kg}$ mass block, which is not the case with the scaled sample. It is expected that the scales would have been crushed due to not being able to sustain the blocks weight. Instead, this sample was attached to a module housing prototype weighing $171.91 \mathrm{~g}$. The difference in weight is not an issue, as any mass difference is accounted for when calculating coefficients of friction.

The flat silicone sample was tested along four substrate surfaces (steel, carpet, acetate, laminate). The skin layer was tested across steel and carpet, with both forwards and backwards motion recorded.

Flat silicone results. From Fig. 7, it appears that the frictional resistances across carpet, acetate and laminate are relatively similar. This suggests that, when the scales are flat against the module housing, they will not hinder the 


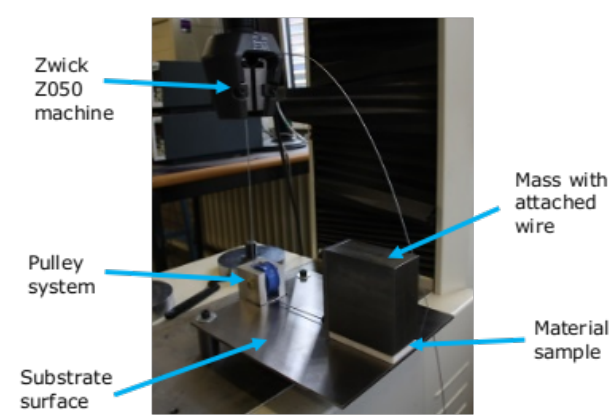

a)

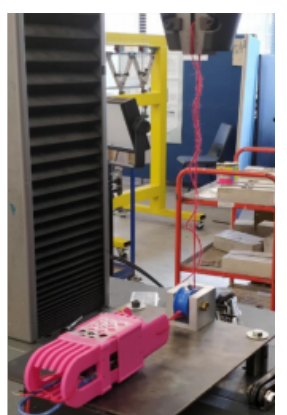

b)

Fig. 6: Frictional testing rig (a) Set-up for the flat silicone sample (b) Set-up for the scaled silicone sample

robots serpentine and sidewinding motion. However, it should be noted that the steel and acetate tests did not produce a stabilised force range during travel.

Scaled silicone results. Although three of the four tested cases did not display a consistent magnitude of frictional force during motion, there are meaningful findings with regards to the impact of the scale direction on motion. For the steel surface, there is more resistance to the scales in the forwards direction than backwards (scale orientation depicted in Fig. 8). This is because, on a flat surface, the scales have nothing to interlock with. They become compliant, increasing contact area with the steel substrate and therefore adhesion-mediated friction becomes the dominant interaction [7]. In the backwards direction, only the points of the scales contact the surface so there is less frictional resistance due to adhesion in this direction.

For the carpet substrate, the opposite relationship is demonstrated, with $\mathrm{k}$ in the backwards direction being larger than in the forwards direction. The scales in the forwards direction grip more effectively with the carpet, with the stiff points propelling the module housing ahead. In the backwards direction, the scale orientation and carpet roughness interlock opposingly, thus preventing smooth travel. This may explain the jagged nature of the frictional force data recorded in Fig. 7.
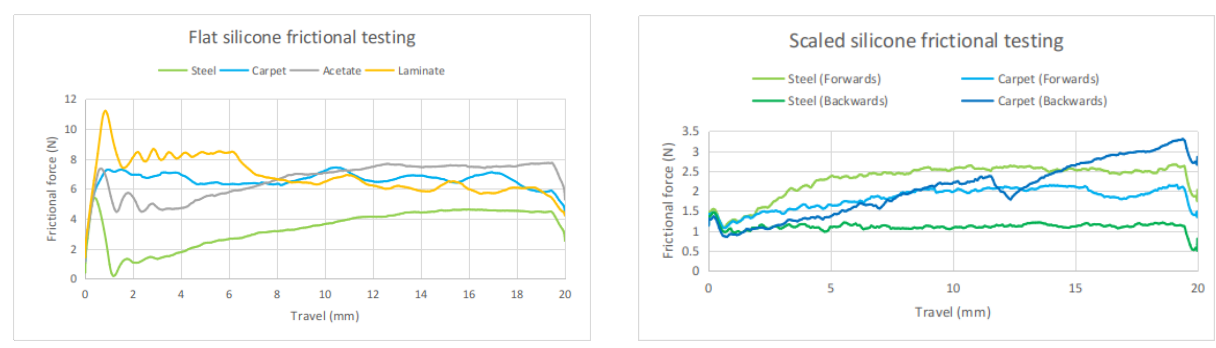

Fig. 7: Frictional testing results for flat silicone and scaled silicone samples across various substrates 


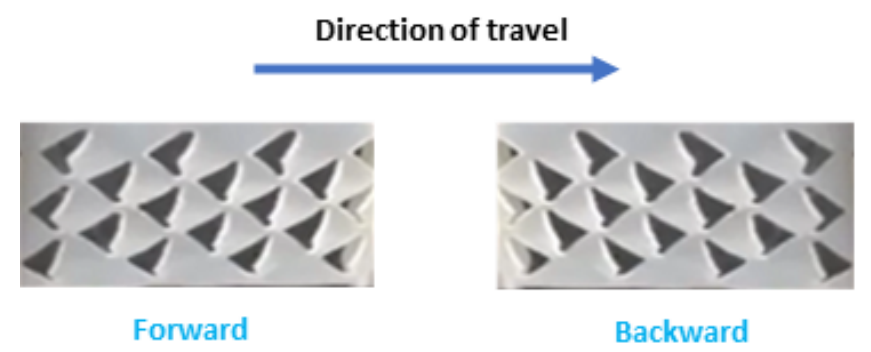

Fig. 8: Scale orientation in the forwards and backwards directions.

\subsection{Prototype 2 - Housing and Smart Servos}

Smart Servo testing. We implemented 5 motions for the robot snake: forwards serpentine, backwards serpentine, left sidewinding, right sidewinding, clockwise rotation, and anticlockwise rotation. The aim of this testing stage is to observe these different motions performed by the robotic snake in both the standard and custom SLS brackets and identify associated issues. The hierarchy of potential issues includes ease of assembly, mechanical movement, motion stability, and bracket durability.

The housing enables the full function of the robot without restriction, enabling the full rotation of servo motors at each bracket whilst providing the rigidity necessary for controlled motion. Initially a 5-motor snake robot was used to test the 6 motions using the standard manufacturer motor brackets. The bracket assembly, motion stability, and mechanical movement of the 6 motions demonstrated no issues with the initial 5-motor prototype.

Backwards serpentine is considerably slower than forwards serpentine, as this movement relies on the tail contacting the ground and pushing off from it. Left and right sidewinding movements have some stability issues, the increased weight and length of the SLS motor brackets means that more weight is shifted onto the mid-section of the snake, causing the robot snake to turn onto its side. It is still able to achieve the desired direction of movement, but with less efficiency.

Initial testing, performed upon the assembly of the prototype, showed that target joint angles greater than 45 degrees produce smoother forwards and backwards serpentine motions. An angle of 45 degrees was originally chosen as it was observed to cause certain sections to not be in contact with the ground to assist the different motions. Angles of 55 degrees and 60 degrees showed smoother movements; these greater angles allowed the "tail" end of the snake to contact the ground and push off. It is also noted that angles lower than 45 degrees give the sidewinding movements more stability. This has the effect of evening out the weight distribution. Further testing would be required to identify the exact causes within the movement issues identified, and to identify optimum angles and joint speeds for the different motions by assessing their speed and stability. 


\section{Conclusion}

This project endeavoured to construct a snake-inspired robot based on the principles of a modular robotic system. Using modular robotics as a base, identical housing units were developed and interlinked to produce a housing chain with alternating degrees of freedom producing rotational and collinear movement. Software was created to incorporate servo motor control and produce serpentine and sidewinding motion. A segregated system was packed into the housing to control the linear actuators to facilitate rectilinear motion. An extendible elastomer skin layer was also manufactured with retractable scales to exclusively facilitate the rectilinear motion of the robot. Two prototypes were assembled, including 1) prototype 1, used to test the snakeskin, demonstrating a single actuator's ability to extend and contract a module housing with a scaled skin layer fixed on, shifting the module housing forwards and 2) prototype 2, used to test the smart servos, demonstrating the motors' ability to allow a housing chain of 5 modules to perform the serpentine and sidewinding motions.

\section{References}

1. Abdel-Aal, H.A.: Surface structure and tribology of legless squamate reptiles. Journal of the Mechanical Behavior of Biomedical Materials 79, 354-398 (2018)

2. Li, M., Cao, Z., Zhang, D., Fu, Y.: 3-dof bionic parallel mechanism design and analysis for a snake-like robot. In: 2016 IEEE International Conference on Robotics and Biomimetics (ROBIO). pp. 25-30 (2016)

3. LISSMANN, H.W.: Rectilinear Locomotion in a Snake (Boa Occidentalis). Journal of Experimental Biology 26(4), 368-379 (02 1950)

4. Rafsanjani, A., Zhang, Y., Liu, B., Rubinstein, S.M., Bertoldi, K.: Kirigami skins make a simple soft actuator crawl. Science Robotics 3(15) (2018)

5. Siciliano, B., Khatib, O. (eds.): Springer Handbook of Robotics. Springer Handbooks, Springer, Berlin, 2 edn. (2016)

6. Ta, T.D., Umedachi, T., Kawahara, Y.: Design of frictional 2d-anisotropy surface for wriggle locomotion of printable soft-bodied robots. In: 2018 IEEE International Conference on Robotics and Automation (ICRA). pp. 6779-6785 (2018)

7. Tramsen, H.T., Gorb, S., Zhang, H., Manoonpong, P., Dai, Z., Heepe, L.: Inversion of friction anisotropy in a bio-inspired asymmetrically structured surface. Journal of The Royal Society Interface 15 (2018)

8. da Veiga, T., Chandler, J.H., Lloyd, P., Pittiglio, G., Wilkinson, N.J., Hoshiar, A.K., Harris, R.A., Valdastri, P.: Challenges of continuum robots in clinical context: a review. Progress in Biomedical Engineering 2(3), 032003 (aug 2020)

9. Whitman, J., Zevallos, N., Travers, M., Choset, H.: Snake robot urban search after the 2017 mexico city earthquake. In: 2018 IEEE International Symposium on Safety, Security, and Rescue Robotics (SSRR). pp. 1-6 (2018)

10. Yamada, H., Hirose, S.: Study on the 3d shape of active cord mechanism. In: Proceedings 2006 IEEE International Conference on Robotics and Automation, 2006. ICRA 2006. pp. 2890-2895 (2006) 